1

2

3

4

5

6

\section{A labeled-line for cold from the periphery to the parabrachial nucleus}

\author{
Junichi Hachisuka $^{1}$, H. Richard Koerber ${ }^{1 *}$ and Sarah E. Ross ${ }^{1^{*}}$
}

${ }^{1}$ Department of Neurobiology and the Pittsburgh Center for Pain Research, University of Pittsburgh, 200 Lothrop St., Pittsburgh, PA, USA

Correspondence: saross@pitt.edu (S.E.R.) and rkoerber@pitt.edu (H.R.K.) 


\section{ABSTRACT}

31 Spinal projection neurons are a major pathway through which somatic stimuli are

32 conveyed to the brain. However, the manner in which this information is coded is poorly

33 understood. Here, we report the identification of a modality-selective spinoparabrachial

34 (SPB) neuron subtype with unique properties. Specifically, we find that cold-selective

35 SPB neurons are differentiated by selective afferent input, reduced neuropeptide

36 sensitivity, distinct physiological properties, small soma size, and low basal drive. In

37 addition, optogenetic experiments reveal that cold-selective SPB neurons are distinctive

38 with respect to their connectivity, with little to no input from either Pdyn or Nos1

39 inhibitory interneurons. Together, these data define a neural substrate supporting a

40 labeled-line for cold from the periphery to the brain.

41

42

43 


\section{INTRODUCTION}

46 Cold, along with heat, pain, itch, and some aspects of touch are conveyed from the spinal

47 cord to the brain via the anterolateral tract (Braz et al., 2014; Todd, 2010). This pathway

48 is made up of neurons that arise from many distinct laminae of the spinal cord and project

49 to numerous regions of the brain, ultimately giving rise to autonomic, affective and

50 discriminative aspects of somatosensation. Although numerous groups have recorded

51 from neurons that contribute to the anterolateral tract, the number of distinct spinal output

52 subtypes remains unclear, and how these parallel channels of information give rise to

53 discrete aspects of somatosensation is not known.

54 To better understand somatosensory coding, it is critical to identify the different

55 channels of output from the spinal cord. In this regard, one of the key challenges is that

56 most spinal output neurons respond to several types of stimuli. For instance, the majority

57 of temperature-responsive spinal output neurons also respond to mechanical input

58 (Allard, 2019; Craig et al., 2001; Craig and Andrew, 2002; Ferrington et al., 1987).

59 Similarly, all spinal output neurons that appear to be tuned for itch also respond to the

60 noxious chemicals mustard oil and capsaicin (Andrew and Craig, 2001; Davidson et al.,

61 2012; Jinks and Carstens, 2002). Finally, wide dynamic range neurons respond to both

62 innocuous and noxious stimuli (Andrew, 2010; Ferrington et al., 1987; Willis et al.,

63 2017). These diverse somatosensory stimuli feel different and engage different autonomic

64 and affective responses, arguing that they must be differentially represented within the

65 nervous system. However, the manner in which this information is encoded by spinal

66 output neurons with polymodal response properties remains a subject of debate.

67 Here, we focused on one aspect of somatosensation - cold - because there was

68 a strong precedent for the idea this modality is conveyed, at least in part, by spinal output 
69 neurons that are modality-selective rather than polymodal. In particular, extracellular

70 recording from cat and monkey spinal cord in vivo has revealed the existence of

71 spinothalamic tract (STT) neurons that respond to cold stimulation to the skin, but not

72 other somatosensory modalities (Craig et al., 2001; Craig and Dostrovsky, 2001; Craig

73 and Hunsley, 1991; Craig and Kniffki, 1985; Dostrovsky and Craig, 1996; Zhang et al.,

74 2006). In rodent, we and others have described spinoparabrachial (SPB) neurons that are

75 cold-selective (Allard, 2019; Andrew, 2009; Hachisuka et al., 2016). In this study, we

76 identified cold-selective output neurons and then further examined whether they show

77 distinctive features indicative of a distinct subtype of SPB neuron. Here, we report that

78 cold-selective SPB neurons are significantly different from other SPB neurons in many

79 regards including neuropeptide responsivity, soma size, physiological character, and

80 microcircuit connectivity. These data suggest that cold is conveyed from the periphery to

81 the parabrachial nucleus via a distinct and specific population of 'SPB-cold' neurons.

\section{RESULTS}

\section{Identification of cold-selective SPB neurons.}

84 In order to characterize the response properties of lamina I SPB neurons to natural

85 stimulation to the skin, we used the semi-intact somatosensory preparation (Koerber and

86 Woodbury, 2002; Mcilwrath et al., 2007) that was recently modified for whole-cell

87 recordings of identified spinal cord neurons (Hachisuka et al., 2016). This preparation

88 comprises of a large portion of spinal cord $(\sim \mathrm{C} 2-\mathrm{S} 3)$, together with L2 and L3 roots,

89 ganglia, saphenous nerve and femoral cutaneous nerve and hind limb skin, dissected in

90 continuum (Figure 1A). Lamina I SPB neurons for whole-cell patch clamp recordings

91 were retrogradely labeled through stereotaxic injections of DiI into the lateral

92 parabrachial nucleus. To functionally characterize these neurons, we measured their 
93 action potentials to cold $\left(0{ }^{\circ} \mathrm{C}\right.$ saline $)$, moderate mechanical stimulation (firm brush

94 and/or von Frey filaments, $1-4 \mathrm{~g})$, and heat $\left(50{ }^{\circ} \mathrm{C}\right.$ saline $)$.

95 In these initial experiments, we recorded from and characterized 34 retrogradely-

96 labeled lamina I SPB neurons. Consistent with previous studies (Allard, 2019; Andrew,

97 2009; Bester et al., 2000; Hachisuka et al., 2016), we found numerous mechano-selective

98 neurons (Figures 1B and D). There was also a large subset of neurons that were

99 polymodal, responding to both mechanical and thermal (Figures 1B, 1E, 1F and $1 \mathrm{G})$.

100 None of these SPB neurons responded to heat alone. In contrast, $\sim 25 \%$ (8 of 34)

101 responded selectively to cold (Figures $1 \mathrm{~B}$ and 1C). Finally, two neurons were considered

102 subthreshold because, although they showed evoked excitatory post synaptic potentials

103 (EPSPs) upon stimulation of the skin, this input was not strong enough to evoke an action

104 potential (Figured 1B and 1H).

105 Cold-selective SPB neurons receive input from one afferent subtype only: cold

106 afferents.

107 Cold-selective SPB neurons are so-defined by their output - they fire in response to cold

108 only. However, whether these cells receive only cold input had never been examined. To

109 examine whether cold-selective SPB neurons receive subthreshold input from other

110 modalities, we recorded evoked EPSCs in response to natural stimulation of the skin. In

111 particular, cold-selective SPB neurons were identified in current clamp mode and then

112 recorded in voltage clamp mode (at the reversal potential of chloride, $-70 \mathrm{mV}$ ) to permit

113 the isolation of excitatory inputs. As expected, application of cold $\left(0{ }^{\circ} \mathrm{C}\right.$ saline $)$ to the

114 skin significantly increased the frequency of excitatory post synaptic currents (EPSCs)

115 onto cold-selective SPB neurons (Figures 2A, 2B and 2C). In contrast, there was no

116 significant increase in the EPSC frequency in response to mechanical stimulation 
117 (Figures 2D, 2E and 2F) or heat (Figures 2G, 2H and 2I). These data suggest that cold-

118 selective SPB neurons receive input from only one modality, cold.

119 Cold-selective neurons are unresponsive or only weakly responsive to Substance P.

120 It has previously been shown that the majority of SPB neurons express Tacr1 (also

121 known as the Neurokinin 1 Receptor), which is the receptor for Substance P (also known

122 as Tac1) (Cameron et al., 2015). We therefore assessed whether cold-selective SPB

123 neurons contained functional Tacr1 to determine whether they belong to this category.

124 Sixteen retrogradely-labeled SPB neurons were studied in voltage clamp mode, and

125 neurons were considered to express functional Tacr1 if an inward current was induced by

126 bath-applied Substance P $(2 \mu \mathrm{M})$. Interestingly, cold-selective SPB neurons showed little

127 to no inward current in response to Substance P (Figures 3A and 3C). In contrast, the

128 majority of other lamina I SBP neurons (i.e., those that were not cold-selective) showed

129 strong inward current to Substance P (Figures 3B and 3C). Overall, the response of cold-

130 selective SPB neurons was significantly smaller than that of other SPB neurons (Figures

$1313 \mathrm{~B}$ and $3 \mathrm{C}$ ), suggesting that cold-selective SPB neurons express little to no functional

132 Tacr1. Since the majority of noxious information is thought to be conveyed by Tacr1-

133 expressing SPB neurons (Doyle and Hunt, 1999; Khasabov et al., 2002; Labrakakis and

134 MacDermott, 2003; Mantyh et al., 1997), this finding supports the idea that nociceptive

135 output and cold output are mediated via distinct channels.

136 Cold-selective SPB neurons have low membrane capacitance, high membrane

137 resistance, and small soma size.

138 To further investigate cold-selective SPB neurons, we examined their passive membrane

139 properties. Cold-selective lamina I SPB neurons were not different than other SPB

140 neurons with respect to resting membrane potential (Figure 4A). However, we found that 
141 cold-selective neurons had significantly lower membrane capacitance (Figure 4B) and

142 higher membrane resistance (Figure 4C). These findings raised the possibility that cold-

143 selective neurons are smaller in size than the others. To address this idea directly, we

144 measured the soma area of SPB neurons that were filled with Alex 488 during recording.

145 In agreement with capacitance and resistance measurements, the soma size of cold-

146 selective SPB neurons was significantly smaller than that of other lamina I SPB neurons

147 (Figures 4D and 4E).

148 Cold-selective SPB neurons are distinct with respect to excitatory input

149 The finding that cold-selective SPB neurons have significantly higher membrane

150 resistance than other SPB neurons raised the possibility that cold-selective neurons have

151 fewer open channels. To address this idea in more detail, we quantified the number and

152 size of spontaneous EPSCs (sEPSCs), recording in voltage clamp mode at a holding

153 potential of $-70 \mathrm{mV}$ to isolate excitatory inputs. All cold-selective SPB neurons showed

154 extremely sparse sEPSCs (Figures 5A and 5B), reflecting a very low level of basal input;

155 in contrast, most of the non-cold selective SPB neurons received a continuous barrage of

156 sEPSCs regardless of their functional response properties (Figures 5A and 5B). Thus, the

157 median sEPSC frequency of cold-selective SPB neurons was significantly lower than that

158 of non-cold neurons (4.1 vs $36.6 \mathrm{~Hz}$ ), whereas the sEPSC amplitude was not different.

159 These findings reinforce the idea that cold-selective SPB neurons are part of a distinct

160 neural circuit that is quiet with respect to ongoing activity.

161 Cold-selective SPB neurons belong to distinct spinal circuits.

162 To further characterize this cold-selective circuit, we examined whether SPB neurons are

163 distinct with respect to the types of inhibitory input they receive. Nos1 and Pdyn

164 populations are two, largely non-overlapping subtypes of inhibitory neurons in the dorsal 
165 horn that are activated by noxious stimuli (Boyle et al., 2017; Iwagaki et al., 2013;

166 Kardon et al., 2014; Polgár et al., 2013; Sardella et al., 2011). In behavioral experiments,

167 Nos1 neurons have been implicated in the inhibition of heat and mechanical stimuli,

168 whereas Dyn neurons have been implicated in the inhibition of itch and mechanical pain

169 (Duan et al., 2014; Huang et al., 2018; Kardon et al., 2014; Ross et al., 2010). Whether

170 either of these inhibitory neuron subtypes plays a role in the inhibition of cold is

171 unknown.

172 To address this, we used double transgenic mice harboring Ai32, a Cre-dependent

173 channel rhodopsin (ChR2), together with either Nos1-CreER or Pdyn-Cre alleles. First,

174 we recorded from ChR2-expressing neurons confirm that optogenetic stimulation with

175 blue light was sufficient to induce action potentials in Nos1 and Pdyn neurons (Figures

$1766 \mathrm{~A}-6 \mathrm{C}$ ). Next, we recorded from SPB neurons in voltage clamp mode (holding potential

$177=-40 \mathrm{mV}$ ) to record optogenically-induced currents that were observed upon activation

178 of either Nos1-CreER or Pdyn-Cre populations (Figure 6D). Somewhat surprisingly, only

179 one of 13 SPB neurons showed evidence of inhibitory input from Nos1-neurons, whereas

18013 of 14 SPB neurons showed IPSCs upon stimulation of Pdyn-Cre neurons (Figure 6E).

181 This finding suggested that, as general rule, SPB neurons receive direct inhibitory input

182 from Pdyn neurons but not Nos1 neurons.

183 To ensure that this apparent absence of synaptic input from Nos1 neurons onto

184 SPB neurons was not simply a technical artifact, we next recorded random lamina I

185 neurons (Figure 6F). We found that 6 of 15 random lamina I neurons showed IPSCs in

186 response to optogenetic stimulation of Nos-CreER neurons (Figures 6F and 6G).

187 Moreover, the IPSC amplitude in random neurons was much stronger (median $=158.7$

$188 \mathrm{pA}, \mathrm{n}=6)$ than that observed in the singe SPB neuron that received input $(20.8 \mathrm{pA}, \mathrm{n}=1)$. 
189 Thus, Nos1 neurons provide functional inhibitory input to some lamina I neurons, but not

190 SPB neurons as a general class.

191 Next, we analyzed the Pdyn input in more detail (Figure 6I). We found that

192 optogenetic stimulation of Pdyn neurons gave rise to large inhibitory post synaptic

193 currents (IPSCs) in most SPB neurons, regardless of their response properties to natural

194 stimulation of the skin (Figure 6E). However, although most cold-selective (5 of 6 ) and

195 all non cold-selective (8 of 8) SPB neurons received IPSCs from Pdyn neurons, there was

196 a significant difference with respect to the magnitude of this inhibition. The amplitude of

197 optogenetically-induced IPSCs in cold-selective SPB neurons was significantly smaller

198 than that observed in other SPB neurons (Figures 6E and 6F). These data suggest that,

199 while Pdyn interneurons provide inhibitory input to many SPB neurons, their contribution

200 to the inhibition of cold-selective SPB neurons is almost negligible.

\section{DISCUSSION}

202 Our study provides evidence that cold is mediated by a subset of SPB neurons that have

203 many distinctive properties including little to no response to Substance P, low

204 capacitance, high resistance, small soma size, low basal drive, and a lack of inhibitory

205 input from either Pdyn or Nos1 inhibitory neurons. Thus, these cells represent a distinct

206 output channel through which cold information is conveyed from the periphery to the

207 brain (Figure 7).

208 The idea that cold is conveyed by a distinct output channel is not without

209 precedent. Previous extracellular single unit recordings in cat, rat and monkey have

210 shown that cold-selective STT neurons are distinct from polymodal nociceptive STT

211 neurons with respect to thalamic projection patterns and conduction velocities (Craig et

212 al., 2002, 2001; Craig and Dostrovsky, 2001; Craig and Hunsley, 1991; Craig and Kniffki, 
213 1985; Dostrovsky and Craig, 1996; Zhang et al., 2006). Our study reveals that the SPB

214 pathway, like the STT pathway, involves a specific channel for cold. Whether cold-

215 selective SPB neurons and cold-selective STT neurons arise from distinct output neurons

216 or from a common subset of spinal projection neurons with divergent collaterals remains

217 to be addressed.

218 One of the intriguing findings of our study is that distinct spinal interneuron

219 subtypes show differential connectivity. Specifically, our optogenetic experiments

220 suggest that most SPB neurons do not receive direct inhibitory input from Nos1 neurons.

221 However, there are likely exceptions to this rule since 'giant cells', which are an

222 extremely sparse population of Tacr1-negative SPB neurons of unknown function, have a

223 very large number of synaptic puncta from Nos1 neurons (Puskár et al., 2001). A second

224 population of inhibitory neurons, Pdyn cells, provides inhibitory input to the majority of

225 SPB neurons in a manner that shows strong modality specificity: some SPB neurons

226 receive strong input, but very little is observed in those that are cold-selective. Since

227 cold-selective SPB neurons have spontaneous IPSCs (data not shown) but receive little to

228 no input from either Nos1 or Pdyn interneurons, this raises the question as to which

229 inhibitory interneuron subtype(s) mediate the inhibition of cold.

230 The results from our study are in good agreement with previous studies and lead

231 to several new predictions. For instance, our study reveals that cold-selective SPB

232 neurons have a relatively small soma size and show fewer EPSCs compared to other

233 subtypes. It has been reported that small SPB neurons have fewer excitatory synapses and

234 express the Gria1 subunit of the AMPA receptor (also known as GluA1), whereas large

235 SPB neurons have numerous excitatory synapses and express Gria4 (also known as

236 GluA4) (Polgár et al., 2010). These observations raise the interesting possibility that 
237 cold-selective SPB neurons express Gria1, rather than Gria4, thereby enabling distinct

238 types of synaptic plasticity. Previous studies have also suggested that cold-selective

239 (albeit random) lamina I neurons in cat are pyramidal (Han et al., 1998), and lamina I

240 pyramidal neurons have been shown to be negative for Tacr1-immunoreactivity in rat and

241 monkey (Almarestani et al., 2007; Saeed et al., 2015; Saeed and Ribeiro-da-Silva, 2013;

242 Yu et al., 2005, 1999). Thus, our finding that cold-selective SPB neurons show little to no

243 response to Substance $\mathrm{P}$ is consistent with the possibility that they may be pyramidal in

244 shape. In future studies, it will be important to perform detailed morphological analyses

245 and comprehensive neurochemical profiling to tests these predictions.

246 We and others have recently shown that cutaneous Trpm8 sensory neurons are

247 cold-responsive neurons with several unique characteristics (Dhaka et al., 2007;

248 Jankowski et al., 2017; Knowlton et al., 2013). For example, as a group they have the

249 fastest conduction velocities of all cutaneous C-fibers, are capable of firing at very high

250 frequencies, and have cell bodies among the smallest in the DRG (Jankowski et al., 2017).

251 However, it should be noted that Trpm8 neurons likely represent more than one subtype

252 of afferent, since $3 / 4$ are unimodal (responding to cold alone), whereas $1 / 4$ are polymodal

253 (responding to mechanical and cold stimuli) (Jankowski et al., 2017). Our data suggest

254 that unimodal Trpm8 afferents are likely those that provide input onto cold-selective SPB

255 neurons. This cold-only pathway, comprising a subset of Trmp8 cells together with cold-

256 selective SPB neurons, forms the cellular basis for true labeled-line for cold from the

257 periphery to the brain.

259 function. Cold-selective afferents have low basal activity and are exquisitely sensitive to

260 rapid changes in temperature (Jankowski et al., 2017). Thus, high frequency activity 
261 within this circuit likely signals cooling. As an event detector, such activity would alert

262 the organism of a shift in its environment, which might be pleasant or aversive,

263 depending on the circumstance. Thereafter, the ongoing activity observed in response to a

264 prolonged cold stimulus might provide temperature information that is critical for

265 thermoregulation. Indeed, SPB neurons are known to regulate body temperature through

266 downstream connections to preoptic area of the hypothalamus (Morrison and Nakamura,

267 2018). Thus, in a cold environment, activity in cold-selective SPN neurons could provide

268 the sensory input that elicits heat production as well as the behavioral drive to seek

269 warmth (Hammel and Pierce, 2003; Nagashima et al., 2000; Romanovsky, 2006).

\section{METHODS}

271 Animals

272 Five- to eight-week-old mice of both sexes were used in this study. Wild type mice

273 (C57BL/6) were purchased from Charles River (Horsham, PA). Genetically modified

274 mice were purchased from The Jackson Laboratory (Bar Harbor ME) and bred in house.

275 These were: Pdyn-IRES-Cre, a non-disruptive Cre recombinase knock-in at the

276 endogenous prodynorphin locus (stock: 027958), Nos1-CreER, a disruptive Cre

277 recombinase knockin to the endogenous neuronal nitric oxide synthase locus (stock:

278 014541), and Ai32, a Cre-dependent ChR2 fusion protein, ChR2(H134)/EYFP inserted

279 into the Rosa locus (stock: 012569). Tamoxifen (Sigma, $0.4 \mathrm{mg} / \mathrm{kg}$; IP) was injected into

280 mice harboring the Nos1-CreER allele at post-natal day 14 (P14), three weeks prior to

281 electrophysiological experiments. Mice were given free access to food and water and

282 housed under standard laboratory conditions. The use of animals was approved by the

283 Institutional Animal Care and Use Committee of the University of Pittsburgh.

\section{Stereotaxic injection of DiI}


285 Four- to six-week-old mice were anesthetized with isoflurane and placed in a stereotaxic

286 apparatus. A small hole was made in the skull bone with a dental drill. A glass pipette

287 was used to inject $100 \mathrm{nl}$ of FAST DiI oil $(2.5 \mathrm{mg} / \mathrm{ml}$; Invitrogen, Carlsbad, CA) into the

288 left lateral parabrachial area (relative to lambda: anteroposterior $-0.5 \mathrm{~mm}$; lateral 1.3

$289 \mathrm{~mm}$; dorsoventral $-2.4 \mathrm{~mm}$ ). The head wound was closed with stitches. After recovery

290 from the anesthesia, the animals fed and drank normally. DiI was injected at least five

291 days prior to electrophysiological recordings.

\section{Semi-intact somatosensory preparation}

293 Semi-intact somatosensory preparation was made as previously described with small

294 modification (Hachisuka et al., 2016). Briefly, young adult mice (5-9 weeks old) were

295 deeply anesthetized and perfused transcardially through the left ventricle with oxygenated

296 (95\% O2 and 5\% CO2) sucrose-based artificial cerebrospinal fluid (ACSF) (in mM; 234

297 sucrose, 2.5 KCl, 0.5 CaCl2, $10 \mathrm{MgSO} 4,1.25 \mathrm{NaH} 2 \mathrm{PO} 4,26 \mathrm{NaHCO} 3,11$ Glucose) at

298 room temperature. Immediately following perfusion, the skin was incised along the

299 dorsal midline and the spinal cord was quickly exposed via dorsal laminectomy. The right

300 hindlimb and spinal cord $(\sim \mathrm{C} 2-\mathrm{S} 6)$ were excised, transferred into Sylgard-lined

301 dissection/recording dish, and submerged in the same sucrose-based ACSF, which

302 circulated at $50 \mathrm{ml} / \mathrm{min}$ to facilitate superfusion of the cord. Next, the skin innervated by

303 the saphenous nerve and the femoral cutaneous nerve was dissected free of surrounding

304 tissue. L2 and L3 DRG were left on the spine. Dural and pial membranes were carefully

305 removed and spinal cord was pinned onto the Sylgard chamber with the right dorsal horn

306 facing upward. Following dissection, the chamber was transferred to the rig. Then the

307 preparation was perfused with normal ACSF solution (in $\mathrm{mM} ; 117 \mathrm{NaCl}, 3.6 \mathrm{KCl}, 2.5$

$308 \mathrm{CaCl} 2,1.2 \mathrm{MgCl} 2,1.2 \mathrm{NaH} 2 \mathrm{PO} 4,25 \mathrm{NaHCO} 3,11$ glucose) saturated with 95\% O2 and 
$3095 \% \mathrm{CO} 2$ at $31{ }^{\circ} \mathrm{C}$. Tissue was rinsed with ACSF for at least 30 min to wash out sucrose.

310 Thereafter, recordings were performed for up to $6 \mathrm{~h}$ post-dissection.

\section{Patch clamp recording from dorsal horn neurons}

312 Neurons were visualized using a fixed stage upright microscope (BX51WI Olympus

313 microscope, Tokyo, Japan) equipped with a 40x water immersion objective, a CCD

314 camera (ORCA-ER Hamamatsu Photonics, Hamamatsu City, Japan) and monitor screen.

315 A narrow beam infrared LED (L850D-06 Marubeni, Tokyo, Japan, emission peak, 850

$316 \mathrm{~nm}$ ) was positioned outside the solution meniscus, as previously described (Hachisuka et

317 al., 2016; Safronov et al., 2007; Szucs et al., 2009). Projection neurons in lamina I were

318 identified by DiI fluorescence following injection into the lateral parabrachial nucleus.

319 Whole-cell patch-clamp recordings were made with a pipette constructed from thin-

320 walled single-filamented borosilicate glass using a microelectrode puller (PC-10;

321 Narishige International, East Meadow NY). Pipette resistances ranged from 6 to $12 \mathrm{M} \Omega$.

322 Electrodes were filled with an intracellular solution containing the following (in $\mathrm{mM}$ ):

$323135 \mathrm{~K}$-gluconate, $5 \mathrm{KCl}, 0.5 \mathrm{CaCl} 2,5$ EGTA, 5 HEPES, $5 \mathrm{MgATP}$, pH 7.2. Alexa fluor

324488 (Invitrogen; $25 \mu \mathrm{M}$ ) was added to confirm recording from the target cell. Signals

325 were acquired with an amplifier (Axopatch 200B, Molecular Devices, Sunnyvale CA).

326 The data were low-pass filtered at $2 \mathrm{kHz}$ and digitized at $10 \mathrm{kHz}$ with an A/D converter

327 (Digidata 1322A, Molecular Devices) and stored using a data acquisition program

328 (Clampex version 10, Molecular Devices). The liquid junction potential was not

329 corrected.

$330 \quad$ Natural stimulation to the skin

331 To search for a cell's receptive field, a firm brush or a $4 \mathrm{~g}$ von Frey filament was applied 332 systematically over the skin. If no response to mechanical stimulation was observed, then 
333 hot $\left(50^{\circ} \mathrm{C}\right)$ or cold $\left(0^{\circ} \mathrm{C}\right)$ saline was applied in pseudorandom order across the skin.

334 Once a receptive field was located, stimuli were reapplied directly to the receptive field

335 for $1 \mathrm{~s}$. For mechanosensitive neurons, a variety of mechanical stimuli were applied

336 (small firm paintbrush and/or von Frey filaments (1, 2 and $4 \mathrm{~g}$ ), but these data were

337 pooled for in this study for simplicity. Thermal stimulation was applied using $1 \mathrm{ml}$ of hot

$338\left(50^{\circ} \mathrm{C}\right)$ or cold $\left(0^{\circ} \mathrm{C}\right)$ saline applied gently to the receptive field over $1 \mathrm{~s}$ using $10 \mathrm{cc}$

339 syringe and $18 \mathrm{G}$ needle. For current clamp recordings, the action potential frequency

340 was calculated in $1 \mathrm{~s}$ bins. Responses to natural stimuli were considered significant if the

341 number of action potentials during the stimulation period $(1 \mathrm{~s})$ were more than 3 times

342 the standard deviation of the baseline period averaged over preceding $9 \mathrm{~s}$. For voltage

343 clamp recordings, EPSCs were detected by MiniAnalysis (Synaptosoft). EPSC frequency

344 for during the stimulation ( $1 \mathrm{~s})$ was calculated. Baseline values were averaged over $9 \mathrm{~s}$

345 before stimulation.

\section{Quantification of soma size}

347 For quantification of soma size, Alexa 488 filled neurons were imaged using Micro-

348 manager (an open source software program) with a 40x objective. The area of the soma

349 was analyzed in ImageJ, tracing the edge of the soma.

\section{$350 \quad$ Optogenetic stimulation}

351 For optogenetic stimulation, a blue light pulse (GFP filter, centered around $485 \mathrm{~nm}$,

352 Lambda DG-4, Sutter instruments) was applied through the objective (40x) of the

353 microscope for $5 \mathrm{~ms}$ using a shutter that was controlled by Clampex software (Clampex

354 version 10, Molecular Devices). The light power on the sample was $1.3 \mathrm{mWmm}^{-2}$. The

355 peak amplitude of outward current induced by blue light stimulation was measured in

356 voltage clamp mode at $-40 \mathrm{mV}$. 


\section{Pharmacology}

358 The drugs (tetrodotoxin: Tocris, Substance P acetate salt hydrate: Sigma) were dissolved

359 in ACSF and applied by exchanging solutions via a three-way stopcock using a modified

360 chamber adapted for pharmacological experiments that limits drug dilution (Hachisuka et

361 al., 2016). 


\section{CONFLICT OF INTEREST STATEMENT}

364 The authors have no conflict of interest to declare.

\section{ACKNOWLEDGMENTS}

366 We thank Michael S. Gold for helpful comments. Research reported in this publication

367 was supported by the National Institute of Arthritis and Musculoskeletal and Skin

368 Diseases of the National Institutes of Health under Award Number

369 R01AR063772 to S.E. Ross) and the National Institute of Neurological Disorder and

370 Stroke of the National Institutes of Health under Award Number R01 NS096705 to H.R.

371 Koerber.

372

373 


\section{REFERENCES}

Allard J. 2019. Physiological properties of the lamina I spinoparabrachial neurons in the mouse. J Physiol 0:JP277447. doi:10.1113/JP277447

Almarestani L, Waters SM, Krause JE, Bennett GJ, Ribeiro-da-Silva A. 2007. Morphological characterization of spinal cord dorsal horn lamina I neurons projecting to the parabrachial nucleus in the rat. J Comp Neurol 504:287-297.

Andrew D. 2010. Quantitative characterization of low-threshold mechanoreceptor inputs to lamina I spinoparabrachial neurons in the rat. J Physiol 588:117-24. doi:10.1113/jphysiol.2009.181511

Andrew D. 2009. Sensitization of lamina I spinoparabrachial neurons parallels heat hyperalgesia in the chronic constriction injury model of neuropathic pain. J Physiol 587:2005-17. doi:10.1113/jphysiol.2009.170290

Andrew D, Craig AD. 2001. Spinothalamic lamina I neurons selectively sensitive to histamine: a central neural pathway for itch. Nat Neurosci 4:72-77. doi:10.1038/82924

Bester H, Chapman V, Besson JM, Bernard JF. 2000. Physiological properties of the lamina I spinoparabrachial neurons in the rat. J Neurophysiol 83:2239-59. doi:10.1152/jn.2000.83.4.2239

Boyle KA, Gutierrez-Mecinas M, Polgár E, Mooney N, O’Connor E, Furuta T, Watanabe M, Todd AJ. 2017. A quantitative study of neurochemically defined populations of inhibitory interneurons in the superficial dorsal horn of the mouse spinal cord. Neuroscience 363:120-133. doi:10.1016/j.neuroscience.2017.08.044

Braz J, Solorzano C, Wang X, Basbaum A. 2014. Transmitting Pain and Itch Messages: A Contemporary View of the Spinal Cord Circuits that Generate Gate Control. Neuron. doi:10.1016/j.neuron.2014.01.018

Cameron D, Gutierrez-Mecinas M, Gomez-Lima M, Watanabe M, Polgár E, Todd AJ. 2015. The organisation of spinoparabrachial neurons in the mouse. Pain 156:1. doi:10.1097/j.pain.0000000000000270

Craig AD, Andrew D. 2002. Responses of spinothalamic lamina I neurons to repeated brief contact heat stimulation in the cat. J Neurophysiol 87:1902-14. doi:10.1152/jn.00578.2001

Craig AD, Dostrovsky JO. 2001. Differential projections of thermoreceptive and nociceptive lamina I trigeminothalamic and spinothalamic neurons in the cat. $J$ Neurophysiol 86:856-70. doi:10.1152/jn.2001.86.2.856

Craig AD, Hunsley SJ. 1991. Morphine enhances the activity of thermoreceptive coldspecific lamina I spinothalamic neurons in the cat. Brain Res 558:93-97. doi:10.1016/0006-8993(91)90719-C

Craig AD, Kniffki KD. 1985. Spinothalamic lumbosacral lamina I cells responsive to skin and muscle stimulation in the cat. J Physiol 365:197-221.

Craig AD, Krout K, Andrew D. 2001. Quantitative response characteristics of thermoreceptive and nociceptive lamina I spinothalamic neurons in the cat. $J$ 
415

416

417

418

419

420

421

422

423

424

425

426

427

428

429

430

431

432

433

434

435

436

437

438

439

440

441

442

443

444

445

446

447

448

449

450

451

452

453

454

455

Neurophysiol 86:1459-1480.

Craig AD, Zhang ET, Blomqvist A. 2002. Association of spinothalamic lamina I neurons and their ascending axons with calbindin-immunoreactivity in monkey and human. Pain 97:105-15.

Davidson S, Zhang X, Khasabov SG, Moser HR, Honda CN, Simone DA, Giesler GJ. 2012. Pruriceptive spinothalamic tract neurons: physiological properties and projection targets in the primate. J Neurophysiol 108:1711-23. doi:10.1152/jn.00206.2012

Dhaka A, Murray AN, Mathur J, Earley TJ, Petrus MJ, Patapoutian A. 2007. TRPM8 Is Required for Cold Sensation in Mice. Neuron 54:371-378. doi:10.1016/j.neuron.2007.02.024

Dostrovsky JO, Craig AD. 1996. Cooling-specific spinothalamic neurons in the monkey. J Neurophysiol 76:3656-65. doi:10.1152/jn.1996.76.6.3656

Doyle CA, Hunt SP. 1999. A role for spinal lamina I neurokinin-1-positive neurons in cold thermoreception in the rat. Neuroscience 91:723-732. doi:10.1016/S03064522(98)00623-X

Duan B, Cheng L, Bourane S, Britz O, Padilla C, Garcia-Campmany L, Krashes M, Knowlton W, Velasquez T, Ren X, Ross SE, Lowell BB, Wang Y, Goulding M, Ma Q. 2014. Identification of Spinal Circuits Transmitting and Gating Mechanical Pain. Cell 159:1417-1432. doi:10.1016/j.cell.2014.11.003

Ferrington DG, Sorkin LS, Willis WD. 1987. Responses of spinothalamic tract cells in the superficial dorsal horn of the primate lumbar spinal cord. J Physiol 388:681703.

Hachisuka J, Baumbauer KM, Omori Y, Snyder LM, Koerber HR, Ross SE. 2016. Semiintact ex vivo approach to investigate spinal somatosensory circuits. Elife 5:1-19. doi:10.7554/eLife. 22866

Hammel HT, Pierce JB. 2003. Regulation of Internal Body Temperature. Annu Rev Physiol 30:641-710. doi:10.1146/annurev.ph.30.030168.003233

Han ZS, Zhang ET, Craig AD. 1998. Nociceptive and thermoreceptive lamina I neurons are anatomically distinct. Nat Neurosci 1:218-25. doi:10.1038/665

Huang J, Polgár E, Solinski HJ, Mishra SK, Tseng PY, Iwagaki N, Boyle KA, Dickie AC, Kriegbaum MC, Wildner H, Zeilhofer HU, Watanabe M, Riddell JS, Todd AJ, Hoon MA. 2018. Circuit dissection of the role of somatostatin in itch and pain. Nat Neurosci 1-10. doi:10.1038/s41593-018-0119-z

Iwagaki N, Garzillo F, Polgár E, Riddell JS, Todd AJ. 2013. Neurochemical characterisation of lamina II inhibitory interneurons that express GFP in the PrPGFP mouse. Mol Pain 9:56. doi:10.1186/1744-8069-9-56

Jankowski MP, Rau KK, Koerber HR. 2017. Cutaneous TRPM8-expressing sensory afferents are a small population of neurons with unique firing properties. Physiol Rep 5:1-11. doi:10.14814/phy2.13234

Jinks SL, Carstens E. 2002. Responses of superficial dorsal horn neurons to intradermal 
serotonin and other irritants: comparison with scratching behavior. J Neurophysiol 87:1280-9.

Kardon AP, Polgár E, Hachisuka J, Snyder LM, Cameron D, Savage S, Cai X, Karnup S, Fan CR, Hemenway GM, Bernard CS, Schwartz ES, Nagase H, Schwarzer C, Watanabe M, Furuta T, Kaneko T, Koerber HR, Todd AJ, Ross SE. 2014. Dynorphin acts as a neuromodulator to inhibit itch in the dorsal horn of the spinal cord. Neuron 82:573-86. doi:10.1016/j.neuron.2014.02.046

Khasabov SG, Rogers SD, Ghilardi JR, Peters CM, Mantyh PW, Simone DA. 2002. Spinal neurons that possess the substance $\mathrm{P}$ receptor are required for the development of central sensitization. J Neurosci 22:9086-98.

Knowlton WM, Palkar R, Lippoldt EK, McCoy DD, Baluch F, Chen J, McKemy DD. 2013. A sensory-labeled line for cold: TRPM8-expressing sensory neurons define the cellular basis for cold, cold pain, and cooling-mediated analgesia. J Neurosci 33:2837-48. doi:10.1523/JNEUROSCI.1943-12.2013

Koerber HR, Woodbury CJ. 2002. Comprehensive phenotyping of sensory neurons using an ex vivo somatosensory system. Physiol Behav 77:589-594. doi:10.1016/S00319384(02)00904-6

Labrakakis C, MacDermott AB. 2003. Neurokinin receptor 1-expressing spinal cord neurons in lamina I and III/ IV of postnatal rats receive inputs from capsaicin sensitive fibers. Neurosci Lett 352:121-124. doi:10.1016/j.neulet.2003.08.042

Mantyh PW, Rogers SD, Honore P, Allen BJ, Ghilardi JR, Li J, Daughters RS, Lappi DA, Wiley RG, Simone DA. 1997. Inhibition of hyperalgesia by ablation of lamina I spinal neurons expressing the substance P receptor. Science 278:275-9. doi:10.1126/science.278.5336.275

Mcilwrath SL, Lawson JJ, Anderson CE, Albers KM, Koerber HR. 2007. Overexpression of neurotrophin-3 enhances the mechanical response properties of slowly adapting type 1 afferents and myelinated nociceptors. Eur J Neurosci 26:1801-1812. doi:10.1111/j.1460-9568.2007.05821.X

Morrison SF, Nakamura K. 2018. Central Mechanisms for Thermoregulation. Annu Rev Physiol 81. doi:10.1146/annurev-physiol-020518-114546

Nagashima K, Nakai S, Tanaka M, Kanosue K. 2000. Neuronal circuitries involved in thermoregulation. Auton Neurosci Basic Clin 85:18-25. doi:10.1016/S15660702(00)00216-2

Polgár E, Al Ghamdi KS, Todd AJ. 2010. Two populations of neurokinin 1 receptorexpressing projection neurons in lamina I of the rat spinal cord that differ in AMPA receptor subunit composition and density of excitatory synaptic input. Neuroscience 167:1192-1204. doi:10.1016/j.neuroscience.2010.03.028

Polgár E, Sardella TCP, Tiong SYX, Locke S, Watanabe M, Todd AJ. 2013. Functional differences between neurochemically defined populations of inhibitory interneurons in the rat spinal dorsal horn. Pain 154:2606-2615. doi:10.1016/j.pain.2013.05.001

Puskár Z, Polgár E, Todd AJ. 2001. A population of large lamina I projection neurons with selective inhibitory input in rat spinal cord. Neuroscience 102:167-176. 
doi:10.1016/S0306-4522(00)00445-0

499

500

501

502

503

504

505

506

507

508

509

510

511

512

513

514

515

516

517

518

519

520

521

522

523

524

525

526

527

528

529

530

531

532

533

534

535

536

537

538
Romanovsky AA. 2006. Thermoregulation: some concepts have changed. Functional architecture of the thermoregulatory system. Am J Physiol Integr Comp Physiol 292:R37-R46. doi:10.1152/ajpregu.00668.2006

Ross SE, Mardinly AR, McCord AE, Zurawski J, Cohen S, Jung C, Hu L, Mok SI, Shah A, Savner EM, Tolias C, Corfas R, Chen S, Inquimbert P, Xu Y, McInnes RR, Rice FL, Corfas G, Ma Q, Woolf CJ, Greenberg ME. 2010. Loss of inhibitory interneurons in the dorsal spinal cord and elevated itch in Bhlhb5 mutant mice. Neuron 65:886-98. doi:10.1016/j.neuron.2010.02.025

Saeed AW, Pawlowski SA, Ribeiro-da-Silva A. 2015. Limited changes in spinal lamina I dorsal horn neurons following the cytotoxic ablation of non-peptidergic $\mathrm{C}$-fibers. Mol Pain 11:54. doi:10.1186/s12990-015-0060-z

Saeed AW, Ribeiro-da-Silva A. 2013. De novo expression of neurokinin-1 receptors by spinoparabrachial lamina I pyramidal neurons following a peripheral nerve lesion. $J$ Comp Neurol 521:1915-28. doi:10.1002/cne.23267

Safronov B V, Pinto V, Derkach VA. 2007. High-resolution single-cell imaging for functional studies in the whole brain and spinal cord and thick tissue blocks using light-emitting diode illumination. J Neurosci Methods 164:292-8. doi:10.1016/j.jneumeth.2007.05.010

Sardella TCP, Polgár E, Watanabe M, Todd a. J. 2011. A quantitative study of neuronal nitric oxide synthase expression in laminae I-III of the rat spinal dorsal horn. Neuroscience 192:708-720. doi:10.1016/j.neuroscience.2011.07.011

Szucs P, Pinto V, Safronov B V. 2009. Advanced technique of infrared LED imaging of unstained cells and intracellular structures in isolated spinal cord, brainstem, ganglia and cerebellum. J Neurosci Methods 177:369-380. doi:10.1016/j.jneumeth.2008.10.024

Todd AJ. 2010. Neuronal circuitry for pain processing in the dorsal horn. Nat Rev Neurosci 11:823-836. doi:10.1038/nrn2947

Willis WD, Trevino DL, Coulter JD, Maunz RA. 2017. Responses of primate spinothalamic tract neurons to natural stimulation of hindlimb. J Neurophysiol 37:358-372. doi:10.1152/jn.1974.37.2.358

Yu XH, Ribeiro-da-Silva A, Ribeiro Da Silva A, De Koninck Y. 2005. Morphology and neurokinin 1 receptor expression of spinothalamic lamina I neurons in the rat spinal cord. J Comp Neurol 491:56-68. doi:10.1002/cne.20675

Yu XH, Zhang ET, Craig AD, Shigemoto R, Ribeiro-da-Silva A, De Koninck Y. 1999. NK-1 receptor immunoreactivity in distinct morphological types of lamina I neurons of the primate spinal cord. $J$ Neurosci 19:3545-55.

Zhang X, Davidson S, Giesler GJ. 2006. Thermally identified subgroups of marginal zone neurons project to distinct regions of the ventral posterior lateral nucleus in rats. J Neurosci 26:5215-23. doi:10.1523/JNEUROSCI.0701-06.2006 
540

A

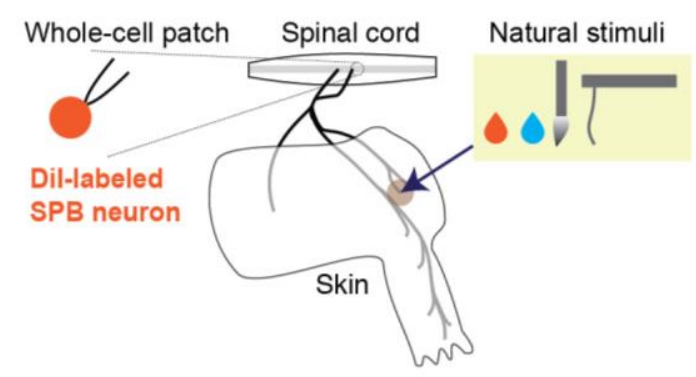

C Cold-selective
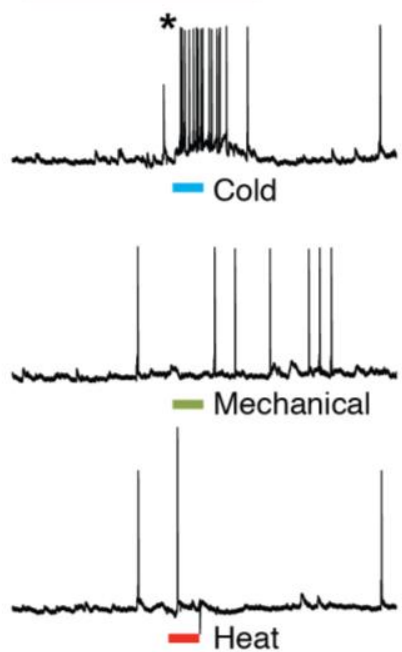

F Mechano- and heat-sensitive
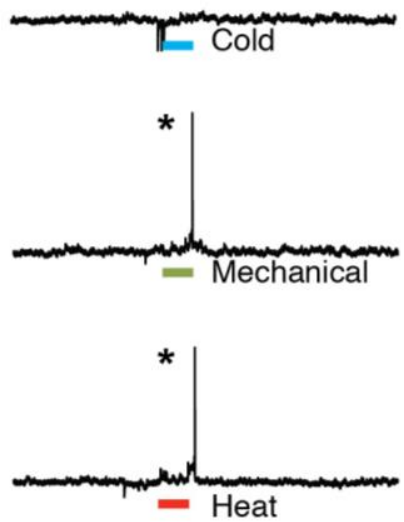

D Mechano-selective
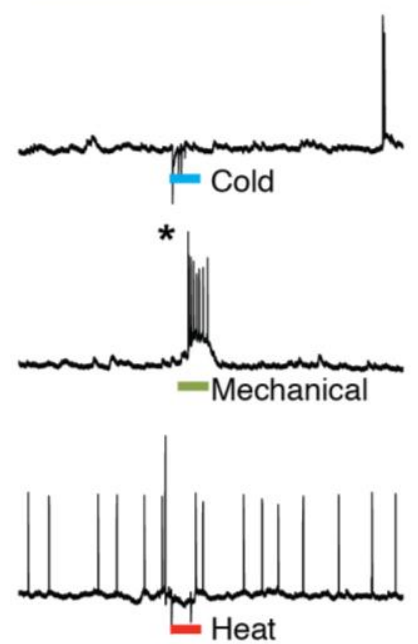

G Mechano-, cold- and heat-sensitive
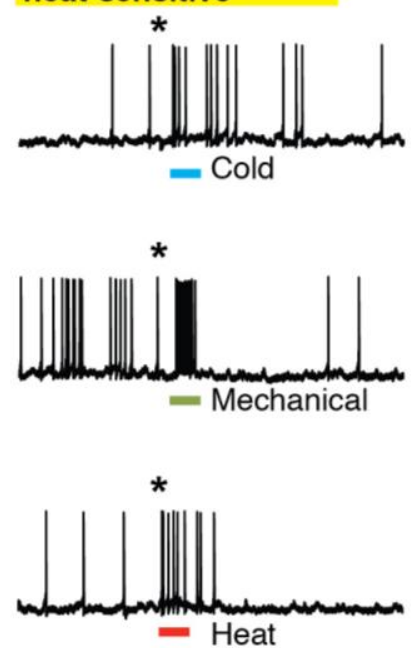

E Mechano- and cold-sensitive
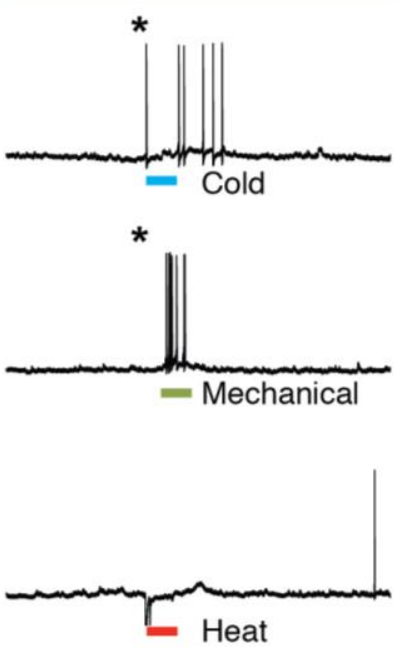

H Subthreshold
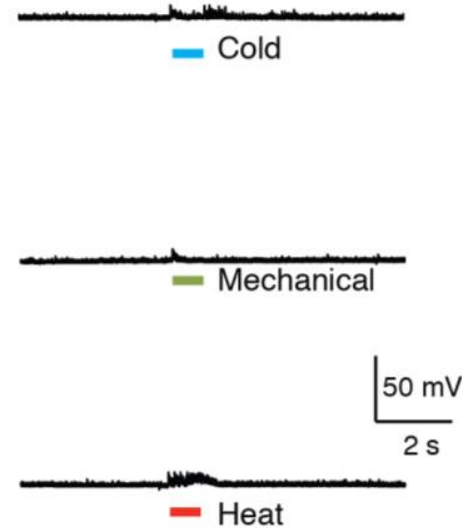

541 


\section{Figure 1. A subset of SPB neurons are cold-selective.}

545 (A) Schematic of the semi-intact somatosensory preparation. Spinal cord, L2 and L3

546 roots, saphenous nerve, lateral femoral cutaneous nerve, and hind paw skin are taken

547 together and whole-cell patch clamp recording is made from retrogradely-labeled SPB

548 neurons. (B) Pie chart of response properties of SPB neurons to mechanical (small, firm

549 brush and/or von Frey filaments, $1-4 \mathrm{~g})$, cold $\left(0^{\circ} \mathrm{C}\right.$ saline $)$ and heat $\left(50{ }^{\circ} \mathrm{C}\right.$ saline $)$

550 stimulation to the skin. Approximately $25 \%$ of SPB neurons are cold-selective. $n=34$

551 SPB neurons. (C-H) Example traces of a cold-selective SPB neuron (C), a mechano-

552 selective neuron (D) a mechano- and cold-sensitive neuron (E), a mechano- and heat-

553 sensitive neuron (F), a mechano- heat- and cold-sensitive neuron (G), and a subthreshold

554 neuron, which showed EPSPs but no action potentials. * indicates response to stimulation

555 is greater than $3 \mathrm{SD}$ of the baseline activity. 
A
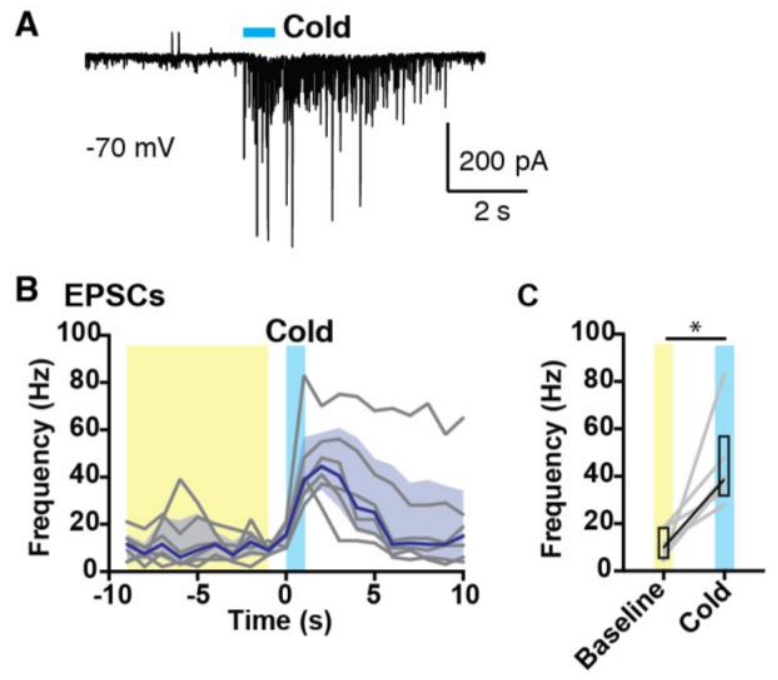

D

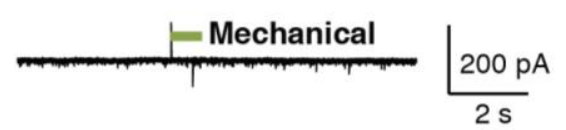

E EPSCs

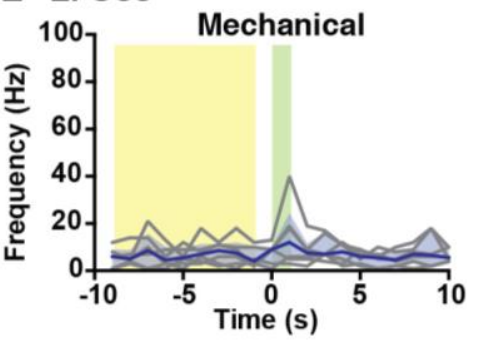

$\mathbf{F}$

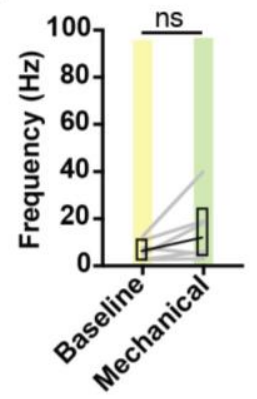

G
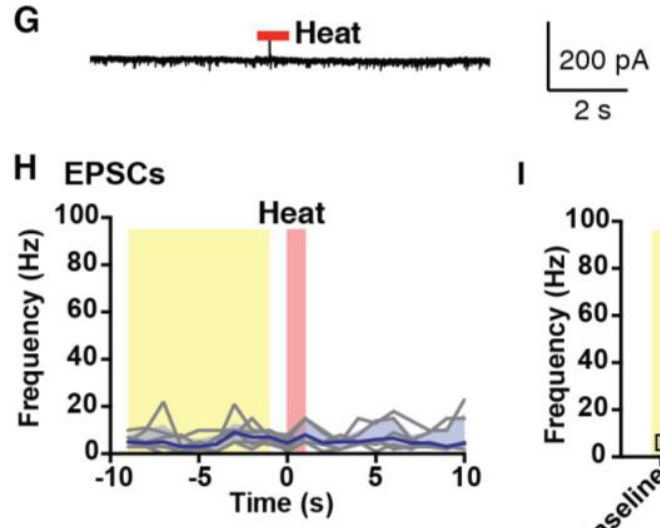
I
Figure 2. Cold-selective SPB neurons receive only cold input.

Analysis of EPSCs of cold-selective neurons evoked by natural stimuli. (A-C)

Representative trace (A) and quantification (BC) of EPSCs observed in response to cold $\left(0^{\circ} \mathrm{C}\right.$ saline). (D-F) Representative trace (D) and quantification (E-F) of EPSCs observed in response to mechanical stimulation (stiff brush or von Frey filaments, 1, 2 or 4 g)). (G-I) Representative trace (G) and quantification (HI) of EPSCs observed in response to heat $\left(0^{\circ} \mathrm{C}\right.$ saline). Black lines are median, shaded area represents interquartile range, and grey lines represent data from individual cells $(n=6$ SPB neurons). * indicates EPSC frequency over baseline period (averaged over $9 \mathrm{~s}$, highlighted in yellow) is significantly different than EPSC frequency in response to cold $(1 \mathrm{~s}$, highlighted in blue), $\mathrm{p}<0.05$, Wilcoxon test. For mechanical (highlighted in green) and heat

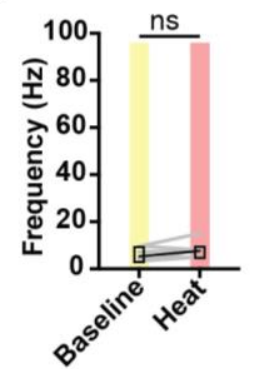

(highlighted in red), n.s. indicates not significant, $\mathrm{p}>0.05$, Wilcoxon test. 
A

Cold-selective

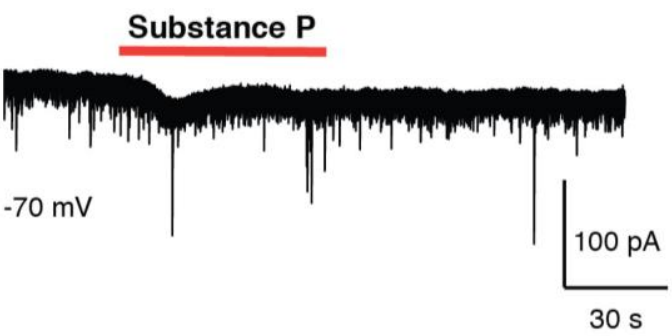

B

Mechano-selective

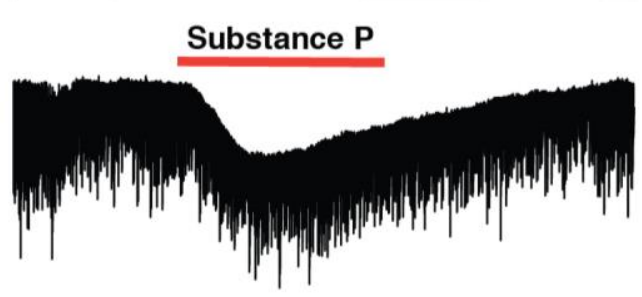

C Substance P Response

581

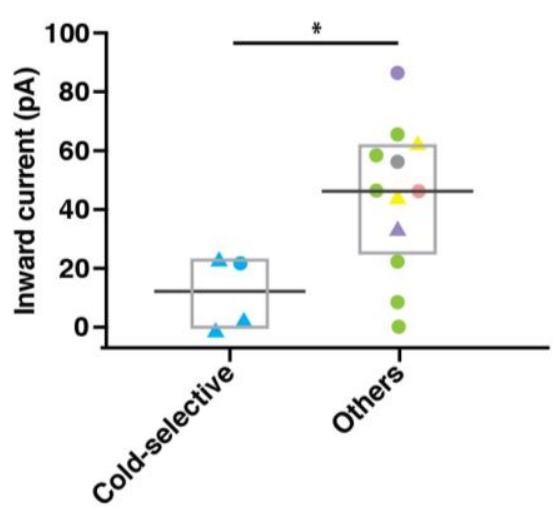

TTX

TTX

$\Delta$ without TTX

- with TTX

- Cold-selective

- Mechano-selective

- Mechano- and cold-sensitive

- Mechano- and heat-sensitive

- Mechano-, heat- and cold-sensitive
- Subthreshold
582

583

584

585

586

587

588

589

590

591

592
Figure 3. Cold-selective SPB neurons show little or no responsive to Substance P.

(A-B) Representative traces of a cold-selective SPB neuron (A) and a mechano-selective SPB neuron (B) in response to bath application of Substance $\mathrm{P}(2 \mu \mathrm{M})$ in the presence of tetrodotoxin $(0.5 \mu \mathrm{M})$. (C) Amplitude of inward current of cold-selective and other SPB neurons induced by Substance P $(2 \mu \mathrm{M})$ in the presence or absence of TTX $(0.5 \mu \mathrm{M})$, as indicated. Since cold-neurons showed little to no response to Substance P even in the absence of TTX, these data were pooled. Box plots are median and interquartile range with symbols representing data points from individual SPB neurons $(n=4$ cold-selective and 12 other SPB neurons, * indicates $\mathrm{p}<0.05$, Wilcoxon test). 
A
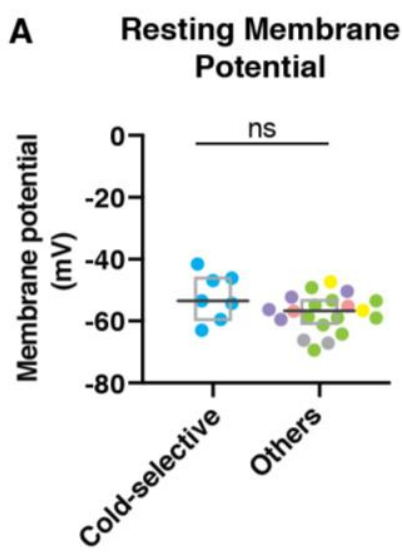

D

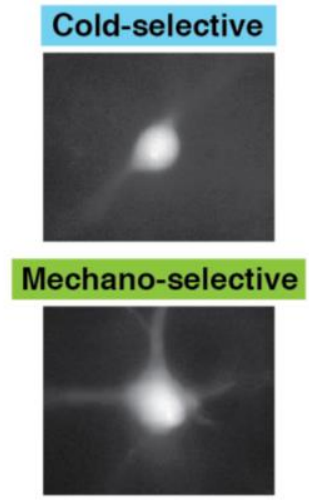

B
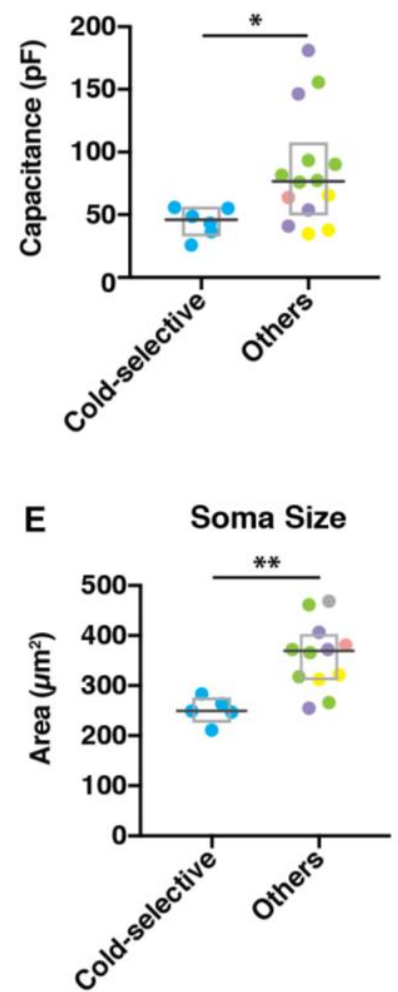

C
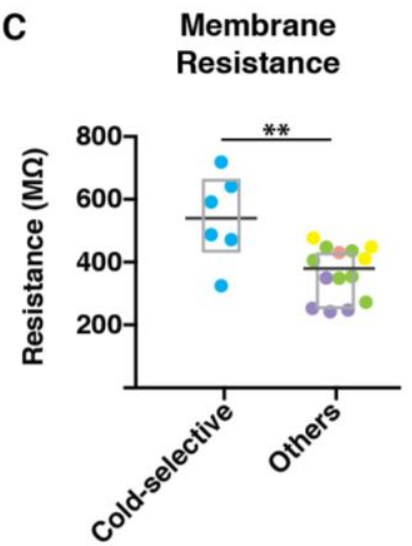

- Cold-selective

- Mechano-selective

- Mechano- and cold-sensitive

- Mechano- and heat-sensitive

Mechano-, heat- and cold-sensitive

- Subthreshold

593

594

595Figure 4. Cold-selective SPB neurons have distinct membrane properties and small soma size.

596(A) Resting membrane potential of cold-selective SPB neurons is not different than other SPB

597 neurons. Box plots are median and interquartile range with symbols representing data points from 598individual SPB neurons $(n=7$ cold-selective SPB neurons and 20 other SPB neurons; n.s., $\mathrm{p}>0.05$, 599Wilcoxon test). (B) Membrane capacitance of cold-selective SPB neurons is significantly lower 600 than that of other SPB neurons. Box plots are median and interquartile range with symbols 601representing data points from individual SPB neurons $(n=6$ cold-selective SPB neurons and 14 602other SPB neurons; * indicates $\mathrm{p}<0.05$, Wilcoxon test). (C) Membrane capacitance of cold603 selective SPB neurons is significantly higher than that of other SPB neurons. Box plots are median 604 and interquartile range with symbols representing data points from individual SPB neurons $(\mathrm{n}=6$ 605 cold-selective SPB neurons and 14 other SPB neurons; $* *$ indicates $p<0.01$, Wilcoxon test). (D-E) 606Representative images (D) and quantification (E) of soma size from cold-selective SPB neurons 607 compared to other SPB neurons. Box plots are median and interquartile range with symbols 608representing data points from individual SPB neurons $(n=5$ cold-selective SPB neurons and 12 609other SPB neurons; ** indicates $\mathrm{p}<0.01$, Wilcoxon test). 
A

Spontaneous EPSCs

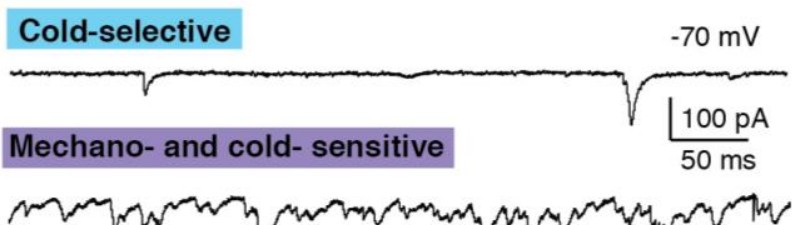

B

sEPSC Frequency

C sEPSC Amplitude
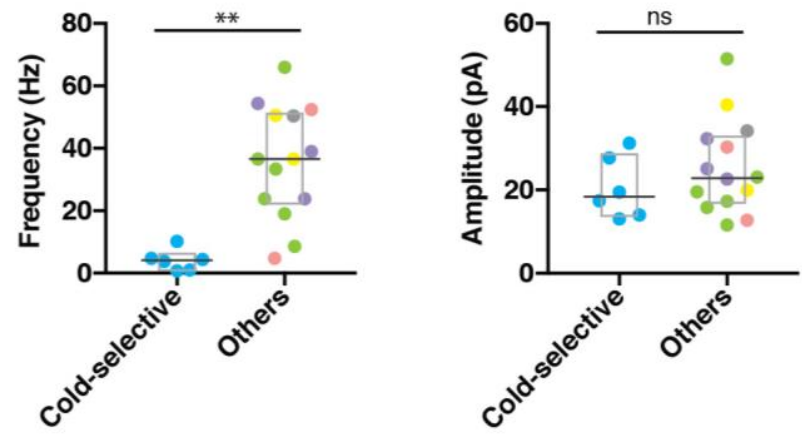

610

- Cold-selective

- Mechano-selective

- Mechano- and cold-sensitive

- Mechano- and heat-sensitive

Mechano-, heat- and cold-sensitive

- Subthreshold

\section{Figure 5. Cold-selective SPB neurons show few EPSCs.}

612 (A) Representative trace of cold-selective SPB neuron (upper trace) and mechano- and

613 cold-sensitive SPB neuron (lower trace). (B-C) Quantification of sEPSC frequency (B)

614 and amplitude (C) of SPB neurons. Box plots are median and interquartile range with

615 symbols representing data points from individual SPB neurons $(n=6$ cold-selective SPB

616 neurons and 14 other SPB neurons; ** indicates $\mathrm{p}<0.01$, n.s. indicates $\mathrm{p}>0.05$

617 Wilcoxon test).

618

619 
A

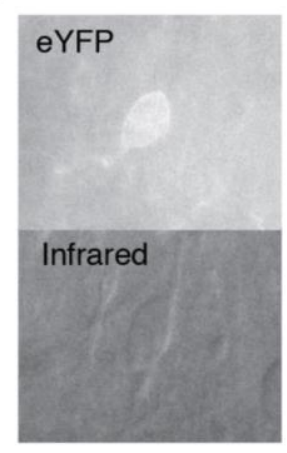

D

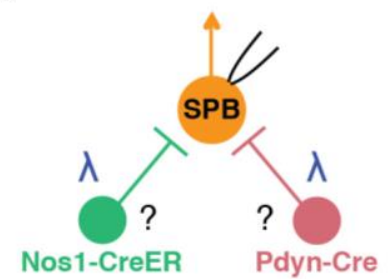

B

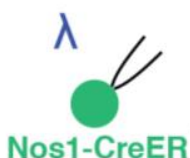
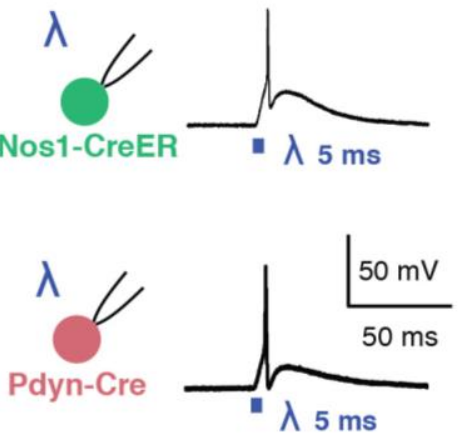

C

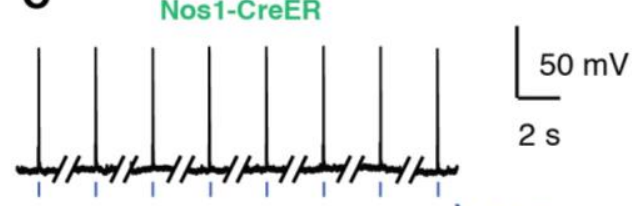

Pdyn-Cre

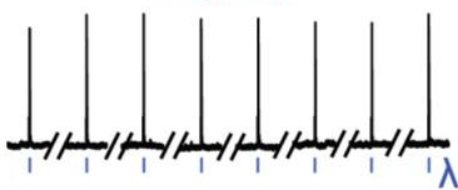

$\lambda 0.1 \mathrm{~Hz}$

E

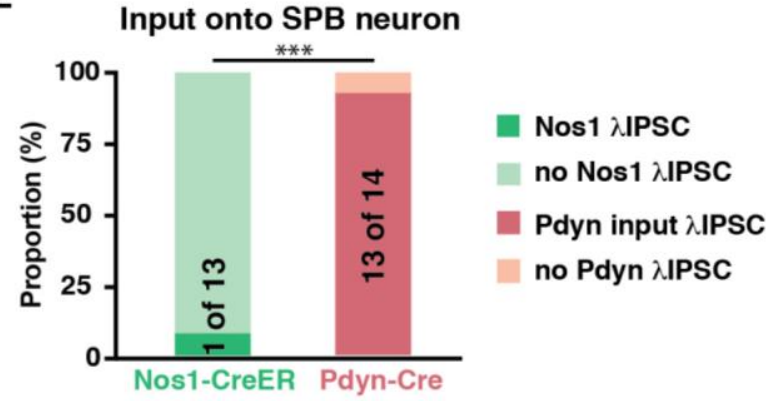

F

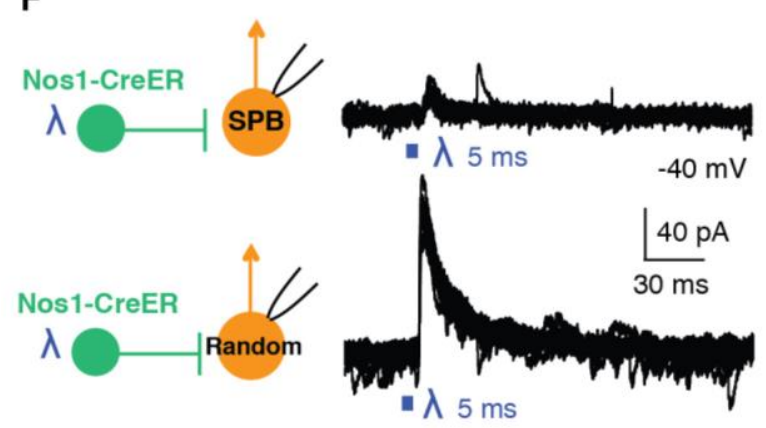

I
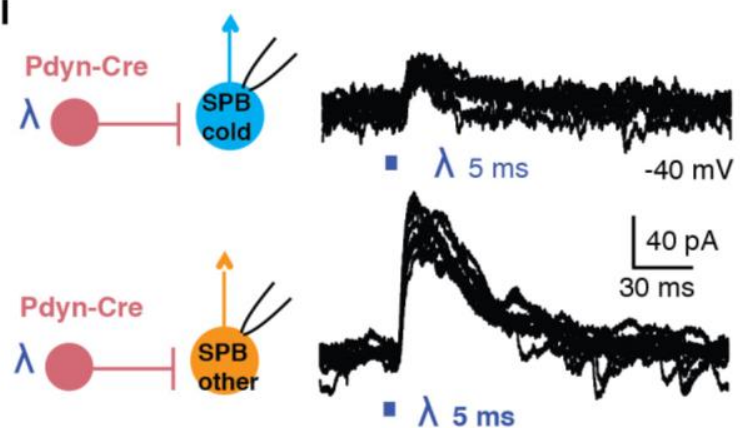

G

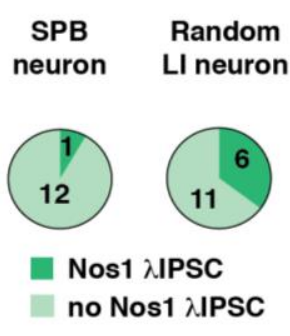

J

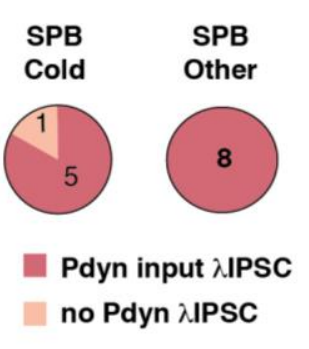

H Nos1 $\lambda$ IPSC Amplitude

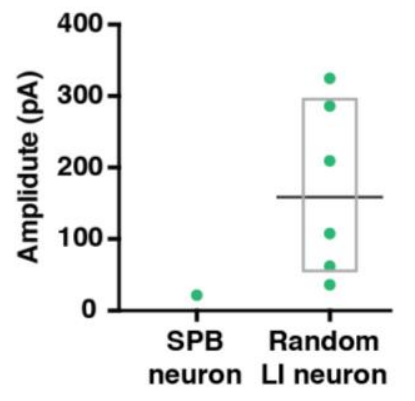

K Pdyn $\lambda$ IPSC Amplitude

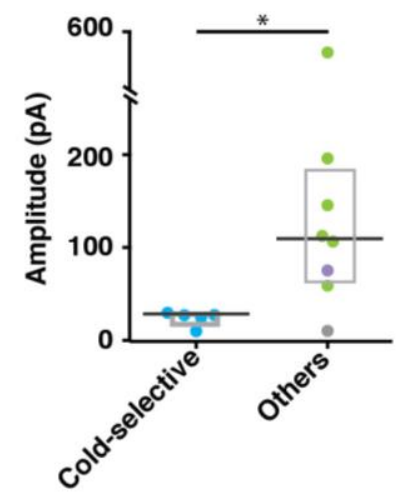


621 Figure 6. Cold-selective SPB neurons belong to distinct spinal circuits.

622 (A) Fluorescent (top) and infrared (bottom) images of a genetically-labeled neuron that is

623 targeted for patch-clamp recordings. (B-C) Optogenetic activation of Nos 1-CreER (top)

624 or Pdyn-Cre (bottom) neurons faithfully elicits action potentials upon single (B) or

625 repeated stimulation at $0.1 \mathrm{~Hz}$ (C). (D) Schematic illustrating experimental design to

626 determine whether SPB neurons receive input from Nos1 or Pdyn inhibitory interneurons.

627 (E) Proportion of SPB neurons that receive IPSCs in response to optogenetic activation

628 of Nos1-CreER (green) or Pdyn-Cre inhibitory interneurons (red). *** indicates

629 significantly different, $\mathrm{p}<0.001$, Fisher's exact test. (F) Schematic and superimposed

630 example traces of IPSCs of a SPB neuron (top) or a random neuron in lamina I (bottom)

631 in response to optogenetic stimulation of Nos-CreER neurons. (G) Proportion of neurons

632 that receive IPSCs in response to optogenetic activation of Nos1-CreER. (H) Amplitude

633 of IPSCs observed upon optogenetic stimulation of Nos1-CreER neurons. Box plots are

634 median and interquartile range (I) Schematic and superimposed example traces of IPSCs

635 of a cold-selective SPB neuron (top) or other SPB neurons (bottom) in response to

636 optogenetic stimulation of Pdyn-Cre neurons. (J) Proportion of SPB neurons that receive

637 IPSCs in response to optogenetic activation of Pdyn-Cre neurons. (K) Amplitude of

638 IPSCs observed upon optogenetic stimulation of Pdyn-Cre neurons. Box plots are median

639 and interquartile range with symbols representing data points from individual SPB

640 neurons ( $\mathrm{n}=5$ cold-selective SPB neurons (blue), 6 mechano-selective neurons (green), 1

641 mechano- and cold-selective (purple) and 1 subthreshold (grey). * indicates significantly

642 different, $\mathrm{p}<0.05$, Wilcoxon test.

643 


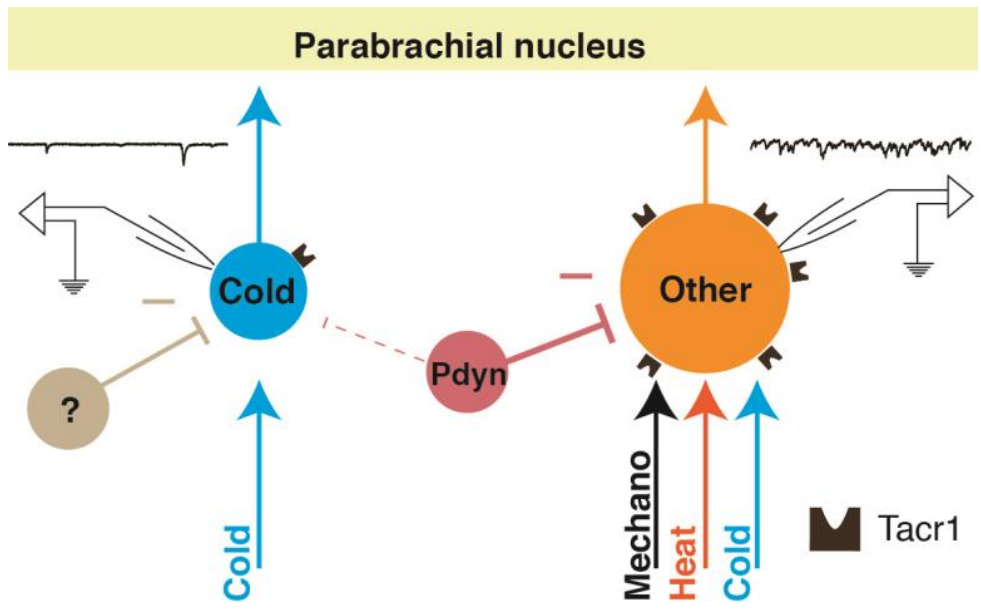

646 Figure 7. A labeled-line for cold from the periphery to the parabrachial nucleus.

647 Cold-selective SPB neurons are distinct from other subtypes in multiple regards including

648 selective input from cold afferents, smaller soma size, little to no expression of Tacr1,

649 low basal drive, and little to no inhibition from Pdyn inhibitory interneurons. 